\title{
BOUNDS ON THE NUMBER OF EDGES OF EDGE-MINIMAL, EDGE-MAXIMAL AND $l$-HYPERTREES
}

\author{
PÉter G.N. Szabó \\ Department of Computer Science and Information Theory \\ Budapest University of Technology and Economics \\ 3-9., Müegyetem rkp., H-1111 Budapest, Hungary \\ e-mail: szape@cs.bme.hu
}

\begin{abstract}
In their paper, Bounds on the number of edges in hypertrees, G.Y. Katona and P.G.N. Szabó introduced a new, natural definition of hypertrees in $k$ uniform hypergraphs and gave lower and upper bounds on the number of edges. They also defined edge-minimal, edge-maximal and $l$-hypertrees and proved an upper bound on the edge number of $l$-hypertrees.

In the present paper, we verify the asymptotic sharpness of the $\left(\begin{array}{c}n \\ k-1\end{array}\right)$ upper bound on the number of edges of $k$-uniform hypertrees given in the above mentioned paper. We also make an improvement on the upper bound of the edge number of 2-hypertrees and give a general extension construction with its consequences.

We give lower and upper bounds on the maximal number of edges of $k$ uniform edge-minimal hypertrees and a lower bound on the number of edges of $k$-uniform edge-maximal hypertrees. In the former case, the sharp upper bound is conjectured to be asymptotically $\frac{1}{k-1}\left(\begin{array}{l}n \\ 2\end{array}\right)$.
\end{abstract}

Keywords: hypertree, chain in hypergraph, edge-minimal hypertree, edgemaximal hypertree, 2-hypertree, Steiner system.

2010 Mathematics Subject Classification: 05C65, 05D99.

\section{REFERENCES}

[1] Z. Baranyai, On the factorization of the complete uniform hypergraph, in: A. Hajnal, R. Rado and V.T. Sós (Eds.), Proceedings of a Colloquium held at Keszthely, June 25-July 1, 1973, Infinite and Finite Sets 1 (North-Holland, Amsterdam, 1975) 91-108.

[2] C. Berge, Hypergraphs (North-Holland, Amsterdam, 1989). 
[3] D. de Caen, Extension of a theorem of Moon and Moser on complete subgraphs, Ars Combin. 16 (1983) 5-10.

[4] H. Hanani, On quadruple systems, Canad. J. Math. 12 (1960) 145-157. doi:10.4153/CJM-1960-013-3

[5] G.Y. Katona and H. Kierstead, Hamiltonian chains in hypergraphs, J. Graph Theory 30 (1999) 205-212. doi:10.1002/(SICI)1097-0118(199903)30:3〈205::AID-JGT5〉3.0.CO;2-O

[6] G.Y. Katona and P.G.N. Szabó, Bounds on the number of edges in hypertrees. arXiv:1404.6430 [math.CO] (2014).

[7] P. Keevash, The existence of designs. arXiv:1401.3665 [math.C0] (2014).

[8] J.X. Lu, An existence theory for resolvable balanced incomplete block designs, Acta Math. Sinica 27 (1984) 458-468.

[9] D.K. Ray-Chaudhuri and R.M. Wilson, The existence of resolvable block designs, in: J.N. Srivastava (Ed.), A Survey of Combinatorial Theory (North-Holland, Amsterdam, 1973) 361-375.

doi:10.1016/b978-0-7204-2262-7.50035-1

Received 4 September 2014

Revised 5 May 2015

Accepted 13 June 2015 